\title{
Using an isomorphic problem pair to learn introductory physics: Transferring from a two-step problem to a three-step problem
}

\begin{abstract}
Shih-Yin Lin and Chandralekha Singh
Department of Physics and Astronomy, University of Pittsburgh, Pittsburgh, Pennsylvania 15213, USA

(Received 2 February 2012; revised manuscript received 5 June 2013; published 10 October 2013)

In this study, we examine introductory physics students' ability to perform analogical reasoning between two isomorphic problems which employ the same underlying physics principles but have different surface features. 382 students from a calculus-based and an algebra-based introductory physics course were administered a quiz in the recitation in which they had to learn from a solved problem provided and take advantage of what they learned from it to solve another isomorphic problem (which we call the quiz problem). The solved problem provided has two subproblems while the quiz problem has three subproblems, which is known from previous research to be challenging for introductory students. In addition to the solved problem, students also received extra scaffolding supports that were intended to help them discern and exploit the underlying similarities of the isomorphic solved and quiz problems. The data analysis suggests that students had great difficulty in transferring what they learned from a two-step problem to a three-step problem. Although most students were able to learn from the solved problem to some extent with the scaffolding provided and invoke the relevant principles in the quiz problem, they were not necessarily able to apply the principles correctly. We also conducted think-aloud interviews with six introductory students in order to understand in depth the difficulties they had and explore strategies to provide better scaffolding. The interviews suggest that students often superficially mapped the principles employed in the solved problem to the quiz problem without necessarily understanding the governing conditions underlying each principle and examining the applicability of the principle in the new situation in an in-depth manner. Findings suggest that more scaffolding is needed to help students in transferring from a two-step problem to a three-step problem and applying the physics principles appropriately. We outline a few possible strategies for future investigation.
\end{abstract}

DOI: 10.1103/PhysRevSTPER.9.020114

PACS numbers: 01.40.gb, 01.40.Ha

\section{INTRODUCTION}

Identifying the relevant physics principles involved is one important component of problem solving in physics. Physics is a subject in which diverse physical phenomena can be explained by just a few basic physics principles. To learn physics effectively, it is essential to unpack the meaning of the abstract principles and understand the applicability of the physics principles in diverse situations [1-9]. One major goal of many physics courses, therefore, is to help students learn to discern the deep similarities between the problems that share the same underlying physics principles but have different surface features, so that students can transfer what they learn from one context to another. However, it is well known that two physics problems that look very similar to a physics expert because both involve the same physics principle do not necessary look similar to the beginning students $[1,10]$. On the other hand, problems that the beginning students consider as similar may actually involve very different physics concepts in the

Published by the American Physical Society under the terms of the Creative Commons Attribution 3.0 License. Further distribution of this work must maintain attribution to the author(s) and the published article's title, journal citation, and DOI. solution steps. For example, a study on the categorization of introductory mechanics problems [1] based upon similarity of solutions indicates that, while experts are likely to place two problems in different categories because one of the problems involves one physics principle (e.g., the principle of conservation of energy) but the other problem involves a different principle (such as Newton's second law), novices may group two problems together because both of them involve an inclined plane. The findings suggest that experts usually group problems based upon the physics principles while novices are more likely to be distracted and group the problems based on the surface features (such as the inclined plane or pulley). The different ways experts and novices categorize problems also reflect the different ways knowledge is organized in their minds [1,2,11-19]. Research suggests that experts in physics have a highly hierarchical knowledge structure, where the most fundamental physics principles are placed at the top, followed by layers of subsidiary knowledge and details $[1,10,20]$. This well-organized knowledge structure facilitates their problem-solving process and helps them approach the problems in a systematic way $[1,2,11-19,21,22]$. It also guides the experts to see the problems beyond the surface features, and makes the transfer of knowledge between different contexts easier. 
As novice students' knowledge structure is usually less organized, it will be beneficial if the instruction provided can help them construct a robust, well-organized knowledge hierarchy (e.g., by learning to extract the deep connections between problems that share the same underlying physics concepts) and help them to understand the broad applicability of the overarching physics principle in various contexts.

Helping students apply what they have learned in one situation to a different situation is an important goal of education. Therefore, a lot of research efforts have been devoted to investigating transfer of learning. In these investigations, issues about transfer of learning have been widely discussed from different perspectives [19,23-31]. For example, the degree to which students can apply knowledge flexibly [23,26,29,32-37], learning features that affect transfer [37-42], and the possible framework to characterize transfer [26-30,33-35,41,43] are discussed in various research contexts. It is pointed out that the amount of knowledge a person has, the knowledge structure that the person constructs, and the context in which the knowledge is learned can all affect the person's ability to apply knowledge flexibly [29]. In order to assist students in learning and help them transfer their learning to different contexts, various scaffolding mechanisms can be used. For example, students can be taught to perform analogical reasoning [1,2,37-39,41,44-46] between problems that involve the same underlying physics principles. Studies have shown that using analogy can help improve students' learning and reasoning in many domains [41,47-51]. A good analogy can help people understand an unfamiliar situation more easily by creating a connection between the new and the existing information [52]. Such connection can make the mental processing of new information more efficient by modifying the existing knowledge schemata. It can also make the new information more concrete and easier to comprehend. Analogy has long been an effective strategy adopted by many instructors in the classrooms. It is also a common practice for students to solve new problems by first looking for similar problems that they already know how to solve and applying similar reasoning strategies from one problem to another. To help students recognize the applicability of a physics principle in various contexts by performing analogical problem solving, students can be explicitly guided to point out the similarities between two problems that involve different surface features but the same underlying physics and take advantage of what they learn from one problem to solve the other. In doing so, students may develop an important skill shared by experts: the ability to transfer from one context to another, based upon shared deep similarities.

In this study, we examine students' abilities to learn from worked-out examples and to perform analogical problem solving between two isomorphic problems. In particular, we investigate whether or not students could discern the similarities between a solved problem and a quiz problem, take advantage of the similarities, and transfer what they learn from the solved problem to solve the quiz problem that is isomorphic. According to the definition from Hayes and Simon [53], problems are isomorphic if they can be mapped to each other in a one-to-one relation in terms of their solutions and the moves in the problemsolving trajectories. For example, the "tower of Hanoi problem" is isomorphic to the "cannibal and the missionary problem" since they have the same structure if reduced to the abstract mathematical form [53]. In our study, we call problems isomorphic if they can be solved using the same physics principles. Research has shown that two problems that are isomorphic are not necessarily perceived as being at the same level of difficulty, especially by a beginning learner $[54,55]$. Depending on a person's expertise in the field, different contexts and representations may trigger the recall of a relevant principle more in one problem than another. Changing the context of the problem, making one problem in the isomorphic pair conceptual and the other quantitative, or introducing distracting features into one of the problems can to different extents raise the difficulty in discerning the similarity and make the transfer of learning between the two problems more challenging [56].

In a prior study [46], we have investigated students' abilities to transfer their learning from a two-step solved problem to a two-step isomorphic quiz problem. In particular, students were explicitly asked in a recitation quiz to browse through and learn from a solved problem (to which a detailed solution was provided) and then use the analogy to solve an isomorphic quiz problem. In that prior study [46], the solved problem provided was about a girl riding a roller coaster on a smooth track. The roller coaster car was initially at rest at a certain height. The problem asked for the apparent weight of the girl as the roller coaster car went over the top of a circular hump given the girl's weight, the radius of the circle, and the heights of different points. The quiz problem, on the other hand, was about a boy on a tire swing created with a rope tied to a tree. Students were asked to find the maximum tension in the rope during the ride given the boy's mass, the length of the rope, and the initial height assuming the boy starts from rest at the initial height. Although these two problems may look very different to a novice student, the solutions to the solved and quiz problems can be matched to each other in a one-to-one manner. Both of them can be solved by decomposing them into two subproblems and applying the principles of conservation of mechanical energy and Newton's second law with centripetal acceleration in each subproblem, respectively. Different types of scaffolding (instructional support) were provided to students in different intervention groups in order to assist students in transferring the knowledge they learned from the solved problem to the quiz problem. Although the quiz problem was challenging, the prior 
study [46] found that with the proper scaffolding support provided, students were able to reason through the analogy between those two problems and performed significantly better on the quiz problem (the tire swing problem) than students who were not provided with the isomorphic solved problem (the roller coaster problem) to learn from.

In this study, students' ability to perform analogical problem solving and transfer their learning from one situation to another between another pair of isomorphic problems is explored. Unlike the previous study [46] in which both the solved and the quiz problems are twostep problems, the quiz problem in this study can be solved by decomposing it into three subproblems while the solved problem requires decomposing it into two subproblems. Since prior research [57] indicated that many students struggle with this three-step quiz problem, the goal in our current study was to examine whether students can benefit from the solved problem and other scaffolding provided, in a case in which the quiz problem involves one more step than the solved problem and can no longer be directly mapped to the solved problem without careful thinking.

\section{METHODOLOGY}

\section{A. Analogical problem-solving activity and the problems}

In this study, students from a calculus-based and an algebra-based introductory physics course were given two isomorphic problems in the recitation quiz. The solution to one of the problems (which we call the "solved problem") was provided. Students were explicitly asked to learn from the solution to the solved problem, point out the similarities between the two problems, and explain whether and how they can exploit the solved problem to solve the other problem (which we call the "quiz problem"). Then they were asked to solve the quiz problem. The solution provided was presented in a detailed and systematic way. It started with a description of the problem with the knowns, unknowns, and target quantity listed, followed by a plan for solving the problem in which the reasons for why each principle was applicable were explicated. After the plan was executed in the mathematical representation, the last part of the solution provided a check for the answer by examining the limiting cases. The solved problem (with its full solution that was provided to the students) and the quiz problem can be found in Appendixes A and B, respectively, of the Supplemental Material [58].

The solved problem was about a boy who took a running start, jumped onto a stationary snowboard, and then went up a hill with the snowboard. The problem asked for the minimum speed at which the boy should run (right before jumping onto the snowboard) in order to go up to a certain height assuming the frictional force can be neglected. The quiz problem, on the other hand, was about two putty spheres hanging on massless strings of equal length. Sphere A was raised to a height $h_{o}$ while keeping the string straight. After it was released, it collided with the other sphere (B), which has the same mass; the two spheres then stuck and swung together to a maximum height $h_{f}$. Students were asked to find $h_{f}$ in terms of $h_{o}$. Both the solved and the quiz problems involve an inelastic collision and process(es) in which something goes up or down while there is no work done by nonconservative forces. Both problems can be solved using the principles of conservation of momentum (CM) and conservation of mechanical energy (CME). However, the snowboard problem can be solved by decomposing it into two steps (first the inelastic collision process, which involves the CM principle, followed by the process of the person and snowboard together going up the hill, which requires the CME principle) while the putty problem involves a three-step solution (with the $\mathrm{CME}, \mathrm{CM}$, and CME principles applicable to the processes of putty A going down, inelastic collision, and putties A and B together going up to a maximum height, respectively). Unlike the previous study [46], in which both the solved and the quiz problems are two-step problems and the solutions can be mapped directly to each other, in this study only the last two steps of the quiz problem and not the whole problem can be mapped directly to the solution of the solved problem. We note that even though the two problems may look very similar to a physics expert and both are relatively easy for them, our previous research indicates that the three-step putty problem is typically very challenging for the introductory students [57]. The investigation in this study was designed to investigate the extent to which providing different types of scaffolding support to students to think about the similarities between the solved problem and the quiz problem may facilitate transfer of what they learned from the two-step solved problem to solve the three-step quiz problem.

\section{B. Participants and the different interventions}

The study involved 180 students from a calculus-based introductory physics course and 202 students from an algebra-based introductory physics course. In each of the courses, students were randomly divided into one comparison group and three intervention groups based on different recitation classes. There was no significant difference between any of the groups in each course in terms of students' Force Concept Inventory (FCI) score conducted at the beginning of the semester.

Students in the comparison group were given only the quiz problem in the recitation quiz. Similar to a traditional quiz, students in this comparison group were asked to solve the quiz problem on their own in 15 minutes; no scaffolding support was provided. The performance of this group of students could help us understand what students were able to do without being explicitly provided a solved isomorphic problem from which to learn. 
Students in the three intervention groups, on the other hand, were given an opportunity to learn from the solved isomorphic problem during the quiz. As research on learning from worked-out examples [59-63] suggests, larger learning gain can be achieved if students are actively engaged in the process of sense making while learning from examples. In order to help students process through the analogy between the two problems deeply and to contemplate issues which they often have difficulty with, the students in different intervention groups were provided with different kinds of scaffolding supports in addition to the solved problem. A summary of the different scaffolding supports implemented in each intervention group is presented in Table I. We will discuss the details and the rationale behind each intervention in the following.

In particular, students in intervention group 1 were asked to take the first 10 minutes in the quiz to learn from the solution to the solved problem (the snowboard problem). They were explicitly told at the beginning of the quiz that after 10 minutes they had to turn in the solution, and then solve two problems in the quiz: one of them would be exactly the same as the one they just browsed over (the snowboard problem), and the other one would be similar (the putty problem). In order to help students discern the connection between the two problems, students were also explicitly asked to identify the similarities between the two problems and explain whether they could use the similarities to solve the quiz problem before actually solving it. The fact that the solution we provided made explicit the consideration for using the principles but was not directly the solution to the quiz problem was inspired by the Schwartz, Bransford, and Sears theory of transfer [43], which states that two components-efficiency and innovation-are both important in the learning and transfer processes. We hypothesized that since students had to solve the same problem they browsed over (i.e., a task toward the efficiency domain) and an isomorphic problem in the quiz (i.e., a task toward the innovation domain), students would try their best to get the most out of the solution in the allocated learning period. In order to apply what they learned from the solution to solve exactly the same problem on their own as well as the isomorphic problem, they had to not only figure out what principles to use, but also understand why and how each principle is applicable in different circumstances. We hypothesized that an advantage could be achieved over the comparison group if students in intervention group 1 went through a deep reasoning while browsing over the solved problem as we intended. Students' performance on both problems was later analyzed.

TABLE I. Summary of the different interventions used in this study.

\begin{tabular}{|c|c|c|}
\hline Group name & Students were asked to ... & $\begin{array}{l}\text { Compared to the interventions used in the } \\
\text { prior study [46]... }\end{array}$ \\
\hline Comparison & $\begin{array}{l}\text { Solve the quiz problem on their own. No solved problem } \\
\text { was provided. }\end{array}$ & The same \\
\hline Intervention 1 & $\begin{array}{l}\text { (a) First learn from the solved problem provided } \\
\text { (b) Return the solution to the solved problem } \\
\text { (c) Solve both the solved and quiz problems }\end{array}$ & The same \\
\hline Intervention 2 & $\begin{array}{l}\text { (a) Solve the quiz problem on their own first } \\
\text { (b) Learn from the solution to the solved problem } \\
\text { (c) Redo the quiz problem a second time (with the solved } \\
\text { problem in their possession) }\end{array}$ & The same \\
\hline Intervention 3 & $\begin{array}{l}\text { Learn from the solution to the solved problem and then } \\
\text { solve the quiz problem (with the solved problem in } \\
\text { their possession). They were also given extra hints } \\
\text { about (a) the fact that similar principles (CM and } \\
\text { CME) can be used to solve both problems and (b) they } \\
\text { might have to use CME twice. } \\
\text { Note: The exact wording for this intervention can be } \\
\text { found in Appendix B in the Supplemental } \\
\text { Material [58]. }\end{array}$ & $\begin{array}{l}\text { The same except that the problem-specific hints (a) and } \\
\text { (b), which are designed based on common student } \\
\text { mistakes on the quiz problem, are modified. } \\
\text { Note that in the prior study [46] students in the inter- } \\
\text { vention group } 3 \text { were asked the following: Learn from } \\
\text { the solution to the solved problem and then solve } \\
\text { the quiz problem (with the solved problem in their } \\
\text { possession). They were also given extra hints about } \\
\text { (a) the fact that similar principles (CME and Newton's } \\
\text { second law with centripetal acceleration involved) can } \\
\text { be used to solve both problems and (b) the implica- } \\
\text { tions of two common conceptions of centripetal force } \\
\text { (one is correct and the other corresponds to a common } \\
\text { student mistake). Students were also guided to select } \\
\text { the concept they agreed with and discuss why. }\end{array}$ \\
\hline
\end{tabular}


The scaffolding in intervention group 2 was designed based on a different framework. Students in this group were first asked to solve the quiz problem on their own. After a designated period of time, they turned in their solutions and were given the isomorphic solved problem to learn from. Then, with the solved problem and its solution in their possession, they were asked to redo the quiz problem a second time after pointing out the similarities between the two problems and were explicitly asked to discuss the implication of these similarities in constructing their solution to the quiz problem. We hypothesized that postponing the browsing over the solved isomorphic problem until the students have actually tried to solve the quiz problem on their own could be beneficial to them because, in this way, students would have already searched through their knowledge base of physics and attempted to organize the information given in the quiz problem. We hypothesized that having tried the quiz problem on their own may make the browsing over the solved problem for relevant information more structured and productive before students attempted the quiz problem a second time. Even if their initial method of solution was incorrect or could not lead them very far, the thinking processes involved may still provide a useful framework for interpreting, incorporating, and accommodating the material that they later learned from the solved problem. We hypothesized that if they got stuck in the first trial without scaffolding, this initial struggle and then browsing over the solved isomorphic problem would give them some perspective on why they were stuck and they may become more deliberate and directed in terms of what to look for in the solution. If they failed to recall a certain principle or forgot to take into consideration a certain part in the problem, the similarity between the two problems may trigger the recall of the previously inaccessible knowledge resource. Moreover, if students were not sure whether their solution was correct, the comparison between the two solutions (one provided, one their own) could also serve as a basis for examining the correctness of their answers. Students had the opportunity to display what they learned from the solved isomorphic problem when they solved the quiz problem a second time.

Unlike the students in intervention groups 1 and 2 who had to figure out the similarities between the two problems themselves, students in intervention group 3 were given a different type of hint in the quiz. They were given both the quiz problem and the solved problem at the same time. In addition to the instruction which asked them to first learn from the solved problem and then exploit the similarity to solve the quiz problem, students were explicitly told that, "Similar to the solved problem, the quiz problem can be solved using conservation of momentum and conservation of mechanical energy." We hypothesized that deliberately pointing out that similar principles should be used in both problems may guide students to focus more on the deep physics principles. Moreover, students in this group were explicitly told that they may have to use the conservation of energy twice because our previous research indicates that it is challenging for students to recognize the three-step nature of the putty problem [57]. The full instruction provided to intervention group 3 can be found in Appendix B in the Supplemental Material [58].

In order to facilitate discussion of our research findings, a comparison between the interventions used in the prior study [46] and our current study are presented in Table I. Except for intervention 3, in which some of the additional instructions provided are problem specific (e.g., the instruction of "applying the CME twice" is designed based on the common student difficulties on the quiz problem that were found in the prior research), the other interventions used in our current study were similar to those used in the prior study [46] in which students were asked to transfer between a pair of isomorphic problems. However, even for interventions 1 and 2, the current study investigates transfer from a two-part problem to a threepart problem unlike the prior study where both the solved and the transfer problems were two-part problems.

\section{Data analysis}

In order to examine the effects of the scaffolding supports, students' performance in the quiz was scored by two researchers on a rubric that was developed by both researchers. We found that the similarities between the solved and quiz (transfer) problems that the students described in the first part of their quiz solution did not provide useful information about their ability to actually solve the quiz problem. Common similarities that the students recognized include the following observations: both problems involve an inelastic collision, the principle of conservation of mechanical energy can be used, etc. However, the students did not necessarily point out how the quiz (transfer) problem can be broken into different subproblems and in which subproblem each principle should be applied. Therefore, in the following discussion, we will only focus on students solutions to the quiz problem.

Students' solutions to the quiz problem were scored using the rubric developed. Summaries of the rubrics for the solved problem and the quiz problem are shown in Tables II and III, respectively. The rubrics consist of two parts based upon the principles required. Different scores were assigned in the solved problem than in the quiz problem because the former involves a two-step solution and the latter involves three steps. The rubrics were designed taking into account the common student difficulties found. An inter-rater reliability of more than $80 \%$ was achieved when two researchers independently scored a sample of 20 students.

Students' performance in different intervention groups was later compared to that in the comparison group. Moreover, in order to examine the effects of interventions 
TABLE II. Summary of the rubric for the solved problem.

\begin{tabular}{|c|c|}
\hline Description & Scores \\
\hline $\begin{array}{l}\text { Conservation of momentum } \\
(5 \text { points })\end{array}$ & $\begin{array}{l}\text { Invoking physics principle: } 3 \text { points (students received a score of either } 3 \text { or } 0 \text {, depending on whether } \\
\text { the physics principle was invoked in the solution) } \\
\text { Applying physics principle: } 2 \text { points (one point was taken off if students made a mistake such as } \\
\text { plugging in the wrong number for the masses) }\end{array}$ \\
\hline $\begin{array}{l}\text { Conservation of mechanical } \\
\text { energy ( } 5 \text { points) }\end{array}$ & $\begin{array}{l}\text { Invoking physics principle: } 3 \text { points (students received a score of either } 3 \text { or } 0 \text {, depending on whether } \\
\text { the physics principle was invoked in the solution) } \\
\text { Applying physics principle: } 2 \text { points [one point was taken off if they made a mistake such as plugging } \\
\text { in the wrong number for height or mistakenly writing the kinetic energy as } \frac{1}{2} m v \text { or } m v^{2} \text {, where } m \\
\text { stands for the mass, } v \text { stands for the speed of the object(s) of interest] }\end{array}$ \\
\hline
\end{tabular}

TABLE III. Summary of the rubric for the quiz problem.

\begin{tabular}{lc}
\hline \hline Description & Scores \\
\hline $\begin{array}{l}\text { Conservation of mechanical } \\
\text { energy in the first and } \\
\text { third subproblems }\end{array}$ & $\begin{array}{c}\text { Invoking physics principle in the first and third subproblems: 2 points (1 point for each subproblem) } \\
\text { (6 points) }\end{array}$ \\
& $\begin{array}{c}\text { Applying physics principle correctly: } 4 \text { points [For each subproblem, students received 2 points if } \\
\text { thessing up with the mass(es) of the sphere(s) or mistakenly writing the kinetic energy as } \frac{1}{2} m v\end{array}$ \\
monservation of momentum & or $m v^{2}$, where $m$ stands for the mass, $v$ stands for the speed of the object(s) of interest.] \\
in the second subproblem & Invoking physics principle in the second subproblem: 1 point \\
(4 points) & Applying physics principle correctly: 1 point \\
\hline \hline
\end{tabular}

on students with different expertise and to evaluate whether the interventions were more successful in helping students at a particular level of expertise, we further classify the students in each course as top, middle, or bottom based on their scores on the final exam. Students in the whole course (not distinguished between different recitation classrooms) were first ranked by their scores on the final exam. About $1 / 3$ of the students were assigned to the top, middle, and bottom groups. The number of students in each case is shown in Table IV. As noted earlier, there was no significant difference between any of the groups in each course in terms of students' FCI scores administered at the beginning of the semester. In order to take into account the possible difference between different recitation classes which may develop as the semester progresses, the overall performance of each intervention group is represented by an unweighted mean of students' performance from the three

TABLE IV. The number of students in each group in the calculus-based course and algebra-based course, where "Comp" stands for "comparison" and "Int" stands for "intervention."

\begin{tabular}{lrccccccc}
\hline \hline & \multicolumn{3}{c}{ Calculus based } & \multicolumn{4}{c}{ Algebra based } \\
Group & Comp & Int 1 & Int 2 & Int 3 & Comp & Int 1 & Int 2 & Int 3 \\
\hline Top & 13 & 13 & 13 & 19 & 10 & 27 & 21 & 15 \\
Middle & 12 & 10 & 10 & 35 & 19 & 11 & 17 & 17 \\
Bottom & 9 & 14 & 12 & 20 & 17 & 8 & 24 & 16 \\
Total & 34 & 37 & 35 & 74 & 46 & 46 & 62 & 48 \\
\hline \hline
\end{tabular}

different levels of expertise [64]. We also compared the students' performance in these algebra-based and calculusbased introductory physics courses with the performance of a group of first-year physics graduate students who were asked to solve the quiz problem on their own without any solved problem provided. The performance of the graduate students can serve as a benchmark for what the undergraduate students can achieve as an upper limit.

\section{Interviews}

In order to obtain an in-depth account of introductory physics students' reasoning while they solved the problem and explore additional strategies that may help them, six students from other introductory physics classes who did not participate in the quiz were recruited for one-on-one interviews. Three of the six students we interviewed were enrolled in an algebra-based introductory mechanics course at the time of the interview; the other three students were enrolled in two different calculus-based mechanics courses. The interviews were conducted in the middle of the semester, after all the relevant topics had been covered in the lectures. All the students recruited for the interviews had a midterm score that fell in the middle of their own introductory physics course, ranging from +6 to -15 points above or below the class averages (which fell between $70 \%$ and $76 \%$ for different courses).

During the interviews, students were asked to learn from the solved snowboard problem provided and solve the isomorphic putty problem given. Similar to the previously 
discussed in-class quiz situation, different students in different interviews received different kinds of interventions, which are listed in Table V. The interviews focused not just on understanding the difficulties students had, but also on examining the additional scaffoldings that may be helpful for the students. Some of the interventions were the same as the interventions used in the quantitative data. Some of the interventions were new in the sense that a slight modification was made to the interventions used in the quantitative data to explore additional strategies to help students. For example, in the interviews with students $\mathrm{E}$ and $\mathrm{F}$, a new problem (the "two-block problem" shown in Appendix C of the Supplemental Material [58]) that is isomorphic to the solved problem and the quiz problem was introduced. This two-block problem, which consists of two steps, an object going down, colliding, sticking, and moving together with another object on the horizontal part of the track, was designed in light of the common student difficulties found in the quantitative data. We will discuss the purpose of this new problem in more detail later.

The audio-recorded interviews were typically $1 / 2$ hour to 1 hour long. They were carried out using a think-aloud protocol, which allowed the researchers to follow and record students' thinking processes. Students were asked to perform the task (whether they were reading the solved problem or trying to solve the quiz problem) while thinking aloud; they were not disturbed during the task. All of the questions were asked to the students after they were completely done with the problem solving to the best of their

TABLE V. The interventions students received in the interview.
Student A (calculus based)

Student B (algebra based)

Student C (calculus based)

Student D (algebra based)

Student E (algebra based)

Student F (calculus based)
Intervention 3

Intervention 2

Intervention 2

Two quiz problems (version 1$)^{\mathrm{a}}$ Two quiz problems (version 2$)^{\mathrm{b}}$
Intervention 3

a(1) The student first learned from the solved snowboard problem provided and then solved another problem about "two blocks colliding" with the solved problem in his hand. This two-block problem can be found in Ref. [58]. (2) The researcher asked clarification questions in order to understand what the student did not make explicit earlier and to better understand their difficulties. The researcher also discussed with the student how to solve the two-block problem correctly. (3) The student was asked to take advantage of what he learned from the previous two problems to solve the putty problem.

(1) The student first learned from the solved snowboard problem provided and then solved the two quiz problems (the twoblock bridging problem and the putty problem) with the solved problem in his hand. (2) The researcher asked clarification questions in order to understand what the student did not make explicit earlier and to better understand their difficulties. The researcher also discussed with the student how to solve the twoblock problem correctly. (3) The student was asked to take advantage of what he learned from the previous two problems and attempted to solve the putty problem a second time. abilities. After students completed the quiz, the researcher would first ask clarification questions in order to understand what they did not make explicit earlier and what their difficulties were. Based on this understanding, the researcher then provided some additional supports (sometimes including the physics knowledge required) to the students in order to help them solve the quiz problem correctly if they had not done so. The researcher also outlined or even demonstrated part of the solutions to the students as needed. After helping students learn how to solve the quiz problem correctly, the researcher invited them to reflect on the learning process they just went through (for example, by asking explicitly what was the thing that helped them figure out how to solve the problem) and asked them to provide some suggestion from the student's perspective on how to improve students' performance on the quiz (transfer) problem. The goal of the students' reflection was to help us identify the possible helpful scaffoldings not only based upon what the researchers observed but also based upon students' reflection of their own learning.

\section{RESULTS AND DISCUSSION}

\section{A. Student performances on the quiz problem}

Table VI shows the different answer categories for the answers graduate students provided when they were asked to solve the quiz problem on their own without scaffolding. The frequencies of each type of answer are listed. Twentythree out of 26 graduate students were able to figure out the three-step nature of the solution even though some of them erroneously used $\frac{1}{2} m v$ instead of $\frac{1}{2} m v^{2}$ to calculate the kinetic energy or made mistakes related to the masses on the two sides of the equation in the third step. Two graduate students incorrectly claimed that the total mechanical energy was conserved throughout (including all the processes), forgetting about the fact that there was an inelastic collision involved in which some mechanical energy will be transformed into other forms of energy when two objects stick together. The principle of CM was not invoked in these two student's solutions. The 26 graduate students on average scored 9.2 out of 10 on the quiz problem when scored using the rubric shown in Table III.

As for the introductory students, Tables VII and VIII present students' average scores on the quiz (transfer) problem in the calculus-based and algebra-based courses, respectively. Because of the instructor's time constraint in the recitation classes, the allotted time for students in intervention group 2 to try the quiz problem on their own before learning from the solved problem was slightly less than the time given to those in the comparison group. Therefore, instead of examining how intervention 2 students' prescaffolding performance compares to that of the comparison group, in these tables we focus only on the performance of students in intervention group 2 after the 
TABLE VI. Graduate students' answer categories and frequencies for the putty problem.

Descriptions of graduate students' answers [in the following equations, $m$ and $v$ stand for the mass and the speed of the object(s) of interest, respectively]

Number of students

Correct three-step solution

$m_{A} g h_{o}=\frac{1}{2} m_{A} v_{A}^{2} \Rightarrow v_{A}=\sqrt{2 g h_{o}}$

$m_{A} v_{A}=\left(m_{A}+m_{B}\right) v_{A+B} \Rightarrow v_{A+B}=\frac{\sqrt{2 g h_{o}}}{2}$

$\frac{1}{2}\left(m_{A}+m_{B}\right) v_{A+B}^{2}=\left(m_{A}+m_{B}\right) g h_{f} \Rightarrow h_{f}=\frac{1}{4} h_{o}$

Correct except that in the third step the student used $m g h=\frac{1}{2} m v$

Correct except that in both the first and third steps the student used $m g h=\frac{1}{2} m v$

Correct except that in the third step the masses on the two sides of the equation are not consistent: $m g h_{f}=\frac{1}{2}(2 m) v_{A+B}^{2}$

$m_{A} g h_{o}=\frac{1}{2} m v^{2}=(m+m) g h_{f}$

$m g h_{o}=2 m g h_{f}$

Both $m_{A} g h_{o}=\frac{1}{2} m_{A} v^{2}=\left(m_{A}+m_{B}\right) g h_{f}$ and the three-step solution [but in the third step the student used $\left.\frac{1}{2}\left(m_{A}+m_{B}\right) v_{A+B}=\left(m_{A}+m_{B}\right) g h_{f}\right]$

TABLE VII. Students' average scores out of 10 on the quiz (transfer) problem in the calculusbased course. Note that the standard deviation in each case is shown in parentheses. The performance of the whole group taken together is represented by an unweighted mean of students' average scores from the top, middle, and bottom categories.

\begin{tabular}{lcccc}
\hline \hline & Comparison & Intervention 1 & Intervention 2 & Intervention 3 \\
\hline Top & $8.2(3.1)$ & $9.2(2.2)$ & $8.4(2.4)$ & $8.2(2.9)$ \\
Middle & $6.8(2.9)$ & $6.1(3.4)$ & $8.4(2.1)$ & $6.9(3.1)$ \\
Bottom & $3.9(3.2)$ & $3.8(3.2)$ & $5.2(3.1)$ & $5.4(3.3)$ \\
Average & 6.3 & 6.4 & 7.3 & 6.8 \\
\hline \hline
\end{tabular}

TABLE VIII. Students' average scores out of 10 on the quiz (transfer) problem in the algebrabased course. Note that the standard deviation in each case is shown in parentheses. The performance of the whole group taken together is represented by an unweighted mean of students' average scores from the top, middle, and bottom categories.

\begin{tabular}{lcccc}
\hline \hline & Comparison & Intervention 1 & Intervention 2 & Intervention 3 \\
\hline Top & $3.8(2.9)$ & $5.3(3.2)$ & $7.3(3.0)$ & $6.2(3.1)$ \\
Middle & $1.9(2.5)$ & $3.3(3.0)$ & $4.2(2.3)$ & $5.3(1.8)$ \\
Bottom & $1.9(1.7)$ & $4.5(3.2)$ & $4.6(2.7)$ & $4.2(1.6)$ \\
Average & 2.5 & 4.4 & 5.4 & 5.2 \\
\hline \hline
\end{tabular}

scaffolding support. As shown in Tables VII and VIII, the average scores of the comparison group students in the two courses indicate that many students had great difficulty with the putty problem. Even though students in the three intervention groups received the solved problem and other scaffolding supports to help them solve the quiz (transfer) problem, their performance did not show great improvement. In the calculus-based course, the comparison group students who solved the quiz problem on their own received an average score of 6.3 out of 10 . The average scores of the three intervention groups were similar. Analysis of variance (ANOVA) indicates that none of the intervention groups in the calculus-based course show a statistically different performance from that of the comparison group. In the algebra-based course, even though the scores went up significantly $(p<0.05)$ from 2.5 (in the comparison group) to 4.4, 5.4, and 5.2 in the three intervention groups, respectively, there is still much room for improvement. It turns out that this problem was challenging for the calculus-based students and even more difficult for the algebra-based students. The $p$ values, which compares the performance of the comparison group students and various intervention group students, are listed in Table IX.

Table X presents intervention 1 students' scores on the isomorphic snowboard problem reproduced immediately after browsing over and returning its solution. Table $\mathrm{X}$ shows that the calculus-based and algebra-based students 
TABLE IX. The $p$ values for the comparison of students' performance between the comparison group (Comp) and different intervention (Int) groups in the calculus-based and algebra-based courses.

\begin{tabular}{lcccccc}
\hline \hline & Comp vs Int 1 & Comp vs Int 2 & Comp vs Int 3 & Int 1 vs Int 2 & Int 1 vs Int 3 & Int 2 vs Int 3 \\
\hline Calculus & 0.994 & 0.772 & 0.976 & 0.597 & 0.882 & 0.894 \\
Algebra & 0.001 & 0.000 & 0.000 & 0.561 & 0.810 & 0.984 \\
\hline \hline
\end{tabular}

TABLE X. Students' average scores out of 10 on the quiz (transfer) problem in the calculus-based course.

\begin{tabular}{lcccc}
\hline \hline & \multicolumn{2}{c}{ Solved problem } & \multicolumn{2}{c}{ Quiz problem } \\
& Calculus & Algebra & Calculus & Algebra \\
\hline Top & 9.9 & 8.8 & 9.2 & 5.3 \\
Middle & 9.9 & 6.8 & 6.1 & 3.3 \\
Bottom & 8.9 & 9.4 & 3.8 & 4.5 \\
All & 9.6 & 8.3 & 6.4 & 4.4 \\
\hline \hline
\end{tabular}

achieved an average score of 9.6 and 8.3 out of 10 , respectively, on the snowboard problem. These results suggest that both algebra-based and calculus-based students were generally good at reproducing the solved problem from which they just learned [65]. However, as shown in Tables VII and VIII, the high score they achieved on the solved problem reproduced did not automatically imply that they had the ability to transfer their learning to the isomorphic quiz problem. On average, the quiz (transfer) problem scores by the same group of students dropped by 3.2 and 3.9 points out of 10 in the calculus-based and algebra-based courses, respectively.

Comparing the results of our study with that of the previous study [46] indicates that it is very difficult for students to extend what they learned from a two-step problem to solve a three-step problem as compared to simply going from a two-step problem to another twostep problem. In the previous study [46] in which students were asked to take advantage of what they learned from the solved roller coaster problem to solve the new tire swing problem, we found that students performed reasonably well in transferring what they learned from the two-step solved problem to solve the two-step quiz problem. For example, in that study, the calculus-based students in intervention group 2 received an average score of 9.1 out of 10 on the quiz problem, which is not only significantly better than the average score (6.8 out of 10) of the comparison group students, who did not receive any scaffolding support, but also higher than the benchmark (8.4) set by the graduate students [46]. However, in this study, even though the same scaffolding support was implemented, students in intervention group 2 did not significantly outperform the comparison group. In fact, none of the intervention group in the calculus-based course was significantly better than the comparison group, nor is the average score in any of the groups comparable to the benchmark set by the graduate students. Although there is different content knowledge involved in these two studies (i.e., Newton's second law in the nonequilibrium situation with a centripetal acceleration involved versus the principle of conservation of momentum), we do not believe the content of conservation of momentum is the major reason that makes the transfer in this study extremely challenging. In fact, since the problems used in the previous study require the application of Newton's second law in the nonequilibrium situation with a centripetal acceleration involved, they may be considered more difficult than the problems involving the principle of conservation of momentum. For example, when the roller coaster problem, tire swing problem, and snowboard problem were used in another study [66,67], it was found that if the target problem was also a two-step problem, students who self-diagnosed their own mistakes in the snowboard problem were capable of transferring their learning to solve an isomorphic two-step problem. Comparably, when the same self-diagnosis task was given, the transfer from the two-step roller coaster problem to the two-step tire swing problem was found to be more difficult. In view of these previous studies, we believe that the great difficulty students had in our study transferring from the snowboard problem to the putty problem lies in the fact that going from a two-step problem to a three-step problem was very challenging for students. Since the putty problem has an additional step compared to the snowboard problem, students cannot correctly solve the putty problem by blindly replicating the procedures shown in the solution to the snowboard problem. Rather, they have to be able to decompose the problem appropriately into temporally separated subproblems based on the appropriate physics principles. In addition, they have to be able to figure out how the different subproblems should be connected. If students do not have a holistic picture of the three-step structure of the problem solution, they are likely to make mistakes.

Examining students' written work from the two introductory physics courses in detail and exploring students' problem-solving performance during the think-aloud interviews further confirm our findings that it was very challenging for students to figure out the three-step nature of the quiz (transfer) problem. Table XI presents the common mistakes that the introductory students made. Table XII presents the corresponding percentage of students who made each mistake listed in Table XI. The percentage of students who display good understanding of the three-step nature of the solution structure (even though they may have made some mistakes in the application details such as 
TABLE XI. Common student approaches to and/or mistakes made in the quiz problem.

\begin{tabular}{ll}
\hline \hline Category & $\begin{array}{l}\text { Description of introductory students' approaches and/or mistakes in each category. Examples of } \\
\text { students' work in each category are also provided. }\end{array}$ \\
\hline
\end{tabular}

No major structural mistake

Students were able to figure out the three-step nature of the putty problem (even though they might have made some mistakes in the application details such as erroneously using $\frac{1}{2} m v$ to calculate the kinetic energy or making mistakes related to the masses on the two sides of the equation).

Example 1: Correct three-step solution:

\section{CM_A: No CM \\ CM_B: Wrong CM}

CME_1: CME across collision

CME_2: Summing up KE and PE from different positions

CME_3: Resembling the solved problem

CME_4: $V$ the same

$$
\begin{aligned}
& m_{A} g h_{o}=\frac{1}{2} m_{A} v_{A}^{2} \Rightarrow v_{A}=\sqrt{2 g h_{o}} \\
& m_{A} v_{A}=\left(m_{A}+m_{B}\right) v_{A+B} \Rightarrow v_{A+B}=\frac{\sqrt{2 g h_{o}}}{2} \\
& \frac{1}{2}\left(m_{A}+m_{B}\right) v_{A+B}^{2}=\left(m_{A}+m_{B}\right) g h_{f} \Rightarrow h_{f}=\frac{1}{4} h_{o}
\end{aligned}
$$

Example 2:

Correct except that in the $3 r d$ step, the student wrote $m g h_{f}=\frac{1}{2}(2 m) v_{A+B}^{2}$ (the masses on the two sides of the equation are not consistent)

Note that in order to be placed in this category students cannot make any of the mistakes described in the following categories.

Did not invoke CM principle in the solution

Invoked CM principle in situations that are clearly incorrect (e.g., from the very beginning to the very end)

Example:

$$
\begin{aligned}
m_{A} v_{A}+m_{B} v_{B} & =\left(m_{A}+m_{B}\right) v_{A+B} \\
0 & =\left(m_{A}+m_{B}\right) v_{A+B}
\end{aligned}
$$

Using conservation of mechanical energy in processes that involve the inelastic collision in between (regardless of whether or not the student invoked the CM principle)

Example 1: $m_{A} g h_{o}=\left(m_{A}+m_{B}\right) g h_{f}$

Example 2: $m_{A} g h_{o}=\frac{1}{2}\left(m_{A}+m_{B}\right) v_{A B}^{2}$

Example 3: $\frac{1}{2} m_{A} v_{A}^{2}=\left(m_{A}+m_{B}\right) g h_{f}$

Example 4: $m_{A} g h_{o}=m_{A} g h_{f}$

(a) Combining several processes into one and summing up the PE in one situation with the KE at another situation, and (b) using conservation of mechanical energy in processes that involve the inelastic collision in between (regardless of whether or not the student invoked the CM principle)

Example 1: $m_{A} g h_{o}+\frac{1}{2} m_{A} v_{A}^{2}=\frac{1}{2}\left(m_{A}+m_{B}\right) v_{f}^{2}+\left(m_{A}+m_{B}\right) g h_{f}$

Example 2: $m g h_{o}+\frac{1}{2} m v_{A}^{2}=(2 m) g h_{f}$

Example 3: $m_{A} g h_{o}=\frac{1}{2}\left(m_{A}+m_{B}\right) v_{f}^{2}+\left(m_{A}+m_{B}\right) g h_{f}$

Example 4: $m g h o+\frac{1}{2} m v_{A+B}^{2}=m g h_{f}$

Note that, as discussed in Fig. 2, since most students did not explicate what their variables (especially the various $v$ 's) in their equations mean, it is possible that a solution like the examples shown here would finally lead to a solution similar to those presented in the previous category (CME_1) if the speed(s) is set to be zero. However, unless an explicit indication that the velocity(ies) is zero is found in their work, all the velocities are assumed to be nonzero, and students' work is categorized in this group, not in group CME_1.

Having solutions very similar to that of the solved problem (i.e., having one CM principle in which the students find that $m_{A} v_{A}=\left(m_{A}+m_{B}\right) v_{A+B}$ and one CME principle in which the students use $\left.\frac{1}{2} m v_{A+B}^{2}=m g h\right)$, but failing to figure out the exact structure to solve the problem.

Example 1: Correctly mapping the solution of the snowboard problem to the last two subproblems of the putty problem (i.e., using $h=h_{f}$ ), but not knowing what to do with the first subproblem that was not included in the solved problem and just leaving it unused.

Example 2: After finding $\frac{m_{A} v_{A}}{2 m_{A}}=v_{A+B}$, incorrectly using $v_{A+B}=\sqrt{2 g h}=\sqrt{2 g\left(h_{o}-h_{f}\right)}$ and coming up with a final answer $h_{f}=h_{o}-\frac{1}{g}\left(\frac{m_{A} v_{A}}{2 m_{A}}\right)^{2}$

Velocity of the sphere is the same right before and right after the collision

Example:

$$
\begin{aligned}
m_{A} g h_{o} & =\frac{1}{2} m_{A} v^{2} \Rightarrow v^{2}=2 g h_{o} \\
\frac{1}{2}\left(m_{A}+m_{B}\right) v^{2} & =\left(m_{A}+m_{B}\right) g h_{f} \\
h_{f} & =\frac{v^{2}}{2 g}=h_{o}
\end{aligned}
$$


TABLE XI. (Continued)

\begin{tabular}{ll}
\hline \hline Category & $\begin{array}{l}\text { Description of introductory students' approaches and/or mistakes in each category. Examples of } \\
\text { students' work in each category are also provided. }\end{array}$ \\
\hline $\begin{array}{l}\text { CME_5: Other structural } \\
\text { mistake }\end{array}$ & Other student mistakes (including but not limited to the following cases): \\
& Example 1: Using $1 D$ kinematics equations instead of CME principle \\
& Example 2: Only writing down some mechanical energy terms or variables separately without writing \\
& down any equation to connect them together \\
& Example 3: Directly answering that $h_{f}=\frac{h_{o}}{2}$ without any solution process provided (or the written \\
& solution process does not lead to the final answer provided) \\
& Example 4: Only some scribbles without a clear solution process \\
\hline \hline
\end{tabular}

using $\frac{1}{2} m v$ for kinetic energy or using the wrong mass on one side of the equation similar to those presented in Table VI) is also listed for comparison. As shown in Table XII, only $44 \%-50 \%$ of the calculus-based students and less than $25 \%$ of the algebra-based students were able to figure out the three-step nature of the quiz problem. Examination of introductory students' work indicates that forgetting to invoke the principle of $\mathrm{CM}$ is one of the most common mistakes they made when no scaffolding was provided, especially in the algebra-based course. $24 \%$ of the calculus-based control group students and $50 \%$ of the algebra-based control group students made this mistake. About half of these students simply related the initial potential energy of putty A (when it is raised to the initial height $h_{o}$ ) to the final potential energy of putty A and B (when both of them reach the maximum height $h_{f}$ ) and came up with an expression $m_{A} g h_{o}=\left(m_{A}+m_{B}\right) g h_{f}$ without considering the process in between. Some other students took into account the intermediate process but still came up with a similar answer $m_{A} g h_{o}=\frac{1}{2} m v^{2}=$ $\left(m_{A}+m_{B}\right) g h_{f}$. (Depending on the student, $m$ and $v$ here could stand for the mass and the speed of putty A right before the collision, or the mass and the speed of both putties together right after the collision.) Examination of students' work in the intervention groups indicates that, even for students who recognize that the CM principle is applicable to the collision process after learning from the solved problem, they might not necessarily understand the implication of applying this principle in their solutions. For example, some of the intervention group students successfully found that the speed of two putty spheres together immediately after the collision would be half of the speed of putty A right before the collision by using CM principle, but they just left it aside after that and did not make use of it later. They resorted to other ideas [e.g., $m_{A} g h_{o}=$ $\left(m_{A}+m_{B}\right) g h_{f}$ ] to come up with the final answer. An example of this type of student work was shown in Fig. 1. The think-aloud interviews suggest that one reason that the students invoked the CM principle initially but did not make use of it later in their solution may be due to the fact that students in general did not systematically come up with a plan for solving the problem before implementing the plan. When solving the quiz problem after browsing over the solved problem, many students first wrote down the principle they "believed" should be used (because the same principle was shown in the given solved problem) and then tried to plug in some variables from the new situation in order to solve for the target variable. However, because

TABLE XII. Students' average scores out of 10 on the quiz (transfer) problem in the calculus-based course. Note that a single student may make both a mistake related to the CM principle and a mistake related to the CME principle. [For example, a student who had a solution $m_{A} g h_{o}=\left(m_{A}+m_{B}\right) g h_{f}$ would be categorized as having made two mistakes: "CM_A: No CM" and "CME_1: CME across collision".] Therefore, the numbers in each column would sum up to $100 \%$ only if neither category A nor category B is counted for a mistake.

\begin{tabular}{|c|c|c|c|c|c|c|c|c|}
\hline & \multicolumn{4}{|c|}{ Calculus-based course } & \multicolumn{4}{|c|}{ Algebra-based course } \\
\hline & Comp & Int 1 & Int 2 & Int 3 & Comp & Int 1 & Int 2 & Int 3 \\
\hline No major structural mistake & 44.1 & 48.6 & 48.6 & 50.0 & 6.5 & 15.2 & 24.4 & 10.4 \\
\hline CM_A: No CM & 23.5 & 29.7 & 11.4 & 16.2 & 50.0 & 28.3 & 14.5 & 10.4 \\
\hline CM_B: Incorrect CM & 14.7 & 2.7 & 0.0 & 8.1 & 6.5 & 2.2 & 0.0 & 0.0 \\
\hline CME_1: CME across collision & 26.5 & 21.6 & 8.6 & 20.3 & 28.3 & 21.7 & 16.1 & 20.8 \\
\hline $\begin{array}{l}\text { CME_2: Summing up KE and PE from } \\
\text { different positions }\end{array}$ & 14.7 & 16.2 & 11.4 & 14.9 & 28.3 & 34.8 & 24.2 & 22.9 \\
\hline CME_3: Resembling the solved problem & 0.0 & 2.7 & 17.1 & 6.8 & 2.2 & 10.9 & 17.7 & 29.2 \\
\hline CME_4: $V$ the same & 5.9 & 0.0 & 2.9 & 2.7 & 0.0 & 0.0 & 0.0 & 0.0 \\
\hline CME_5: Other structural mistakes & 8.8 & 10.8 & 11.4 & 5.4 & 34.8 & 17.4 & 17.7 & 16.7 \\
\hline
\end{tabular}




$$
\begin{aligned}
& m v_{A}=2 m v_{A B} \\
& v_{A B}=\frac{1}{2} v_{A} \\
& K E_{f A}=K E_{i A B} \\
& P E_{i A}=P E_{f A B} \\
& m g h_{o}=2 m g h_{f} \\
& h_{f}=\frac{1}{2} h_{o}
\end{aligned}
$$

FIG. 1. An example of an introductory student's answer to the putty (transfer) problem. Even though the student invoked the CM principle, he did not use this principle to find the final answer.

the solution to the quiz problem had many differences compared to the solved problem, such strategies did not get them too far. After writing down an equation using the CM principle, these students did not know what to do with that equation and did not know how to connect it to the target variable. They just left it aside and started working on a new equation (e.g., the conservation of mechanical energy equation) without coming back and relating it to their original work with the momentum principle.

Another common mistake that students made was invoking the CME principle inappropriately in situations that involve the inelastic collision. In Tables XI and XII, all students placed in category CME_1 ("CME across collision") and category CME_2 ("Summing up KE and PE from different positions," where KE is kinetic energy and $\mathrm{PE}$ is potential energy) had this mistake. As shown in Table XII, this mistake was prevalent in both the control and intervention groups, suggesting that even though students recognized the similarity between the isomorphic problems in terms of the principles involved and invoked the relevant principles, many of them did not understand the situations in which each principle should be applicable. The mistake of treating the mechanical energy as conserved throughout the whole process and coming up with a final answer $h_{f}=\left[M_{a} /\left(M_{a}+M_{b}\right)\right] h_{o}=\frac{1}{2} h_{o}$ (categorized in category CME_1: "CME across collision") is one common type of students' work that involves the mistake of invoking the CME principle in inappropriate situations. Figure 2 presents another example that represents another common type of students' incorrect solution. In Fig. 2, there were two steps involved in the solution: the first step involved the CM principle and the other involved the CME principle. Why the student applied these two principles in the manner he did, however, is not clear. One way of interpreting the student's solution to the transfer problem is to assume that $v_{A}$ and $v_{f}$ stand for the speed of putty A right before the collision and the speed for both putty A and B together immediately after the collision. If this assumption is correct, the student would have applied

$$
\begin{gathered}
\Delta \vec{P}=0 \quad \vec{P}_{f}=\vec{P}_{i} \quad 2 m V_{f}=m V_{A}+m K_{B} \begin{array}{c}
V_{B}=0 \\
V_{f}=\frac{1}{2} V_{A}
\end{array} \\
\Delta E_{m e c}=\frac{1}{2} m V_{A}^{2}+m g h_{o}=\frac{1}{2} 2 m V_{f}^{2}+2 m g h_{f} \\
h_{f}=\frac{\frac{1}{2} m V_{A}^{2}+m g h_{o}-m V_{f}^{2}}{2 m g} \\
h_{f}=\frac{\frac{1}{2} V_{A}^{2}+g h_{o}-\left(\frac{1}{2} V_{A}\right)^{2}}{2 g} \\
h_{f}=\frac{\frac{1}{2} V_{A}^{2}+g h_{\circ}-\frac{1}{4} V_{A}^{2}}{2 g} \\
h_{f}=\frac{\frac{g h_{o}-\frac{1}{2} V_{A}^{2}}{2 g}}{2}
\end{gathered}
$$

FIG. 2. An example of a student's incorrect answer. The situations in which the $\mathrm{CM}$ and $\mathrm{CME}$ principles were applied are not clear.

the principle of $\mathrm{CM}$ correctly to the collision process but made a mistake with the CME part because the student erroneously combined the initial potential energy of putty A (when it was released) with the kinetic energy at a later instance (when putty A reached the bottom) and set it equal to the kinetic energy of putties $\mathrm{A}+\mathrm{B}$ together right after the collision plus the final potential energy of putties $\mathrm{A}+\mathrm{B}$ when they reached the maximum height. The mistake of summing up potential energy and kinetic energy from different instances on one side of the CME equation indicates that the student did not fully understand the meaning of the CME principle and he did not know how to apply it correctly. Figure 3 is another example of a student's solution which shows a similar mistake of mixing up several processes into one and applying the CME principle to an incorrect situation. Similar mistakes were classified in the "CME_2: Summing up KE and PE from different positions" group in Tables XI and XII.

Another possible way to interpret the student's solution in Fig. 2 is to postulate that the student realized he should only combine the potential energy and kinetic energy of a system at the same moment on one side of the equation. In this case, $v_{A}$ and $v_{f}$ would stand for the speed of putty A when it was released and the speed of putty $\mathrm{AB}$ together when they momentarily reached the maximum height $h_{f}$, which would mean that $v_{A}$ and $v_{f}$ should both be zero. The student may then be thinking about the mechanical energy being conserved during the whole process, which can be reduced to the previously described common mistake of setting $m_{A} g h_{o}=\left(m_{A}+m_{B}\right) g h_{f}$. The student, however, 


$$
\begin{gathered}
P E_{1}=m_{A} g h_{o} \quad P E_{f}=m_{A+B} g h_{f} \\
m_{A} g h_{o}+\frac{1}{2} m_{A+B} v_{A+B}^{2}=m_{A+B} g h_{f} \\
P_{1}=P_{f} \\
m_{A} v_{A}+\frac{m_{B} v_{B}=\left(m_{A}+m_{B}\right) v_{A+B}}{v_{A+B}=\frac{m_{A} v_{A}}{m_{A}+m_{B}}} \\
m_{A} g h_{o}+\frac{1}{2} m_{A+B}\left(\frac{m_{A} v_{A}}{m_{A}+m_{B}}\right)=m_{A+B} g h_{f} \\
h_{f}=\frac{m_{A} g h_{o}+\frac{1}{2} m_{A+B}\left(\frac{m_{A} v_{A}}{m_{A}+m_{B}}\right)}{m_{A+B} \cdot g}
\end{gathered}
$$

FIG. 3. Another example of a student's solution in which the student mixed up several processes into one and applied the CME principle to an incorrect situation. Similar mistakes were classified in the "CME_2: Summing up KE and PE from different positions" category in Table XI.

would have invoked the CM principle in an incorrect situation. Figure 4 shows an example of the solution of another student who explicitly claimed that the initial momentum of the system equals the final momentum of the system where all the speeds involved were zero. This mistake suggests that the student did not realize that the $\mathrm{CM}$ principle is applicable only during the collision process and not during the entire process. The percentages of students who explicitly applied the CM principle in an incorrect situation are listed in Table XII (presented in group "CM_B: Wrong CM"). We note that when binning student mistakes into categories in Table XII, when there is an ambiguity in interpreting the meaning of the velocity (ies), all the velocities are assumed to be nonzero unless an explicit indication that the velocity(ies) is zero can be found in their work.

In addition to the mistakes previously mentioned, other common student mistakes include having a solution that resembles the solution to the solved problem but failing to figure out the exact structure to solve the problem, treating the speed of the sphere right after the collision the same as the speed of the sphere right before the collision, etc. Examples of these mistakes are presented in Table XI. We note that Tables XI and XII present the distribution of common student answers or mistakes as shown on the quiz. In addition to those tabulated mistakes, there could be other underlying difficulties that students had, which may have led them to arrive at the answers shown in Tables XI and XII. In the following, we will discuss these possible underlying difficulties. Findings from the interviews that shed light on these issues will be presented.

First, we found that one important reason that the students could not figure out the three-step nature of the problem may have to do with the fact that the students may not have a clear picture in mind about what was going on in the quiz problem and they may not necessarily think through the problem in sufficient depth. For example, in the interviews, we found that most students failed to describe the meaning of each variable in a precise way. When the researcher asked students to explicate what each variable, especially the various $v$ 's mean in the interviews [for example, in the equation $m_{A} v_{A}+m_{B} \nabla_{B}=$ $\left.\left(m_{A}+m_{B}\right) v_{A+B}\right]$, they often answered that $v_{A}$ was the velocity "before collision" and $v_{A+B}$ was the velocity "after collision." It was not easy for them to articulate by themselves which velocity before collision they were talking about (e.g., whether it was the velocity of sphere A "right before the collision" or the velocity of sphere A "at the very beginning when it was released"). It was possible that the students initially did not recognize that in both "before the collision" and "after the collision" there were processes involved in which the speed of the sphere(s) varied with the height. Sometimes it took the researcher some effort to explain to the students that "velocity before or after the collision" could mean many different things since at different heights the velocities were different. As suggested by one student during the reflection, the idea of "snapshots of the putty at different points" was very helpful in solving the problem. However, the student was able

$$
\begin{array}{rl|}
m_{A} v_{A i}+m_{B} v_{B i} & =v_{f A+B}\left(m_{A}+m_{B}\right) \\
m_{A}(0)+m_{B}(0) & =0\left(m_{A}+m_{B}\right) \\
0 & =0 \\
K E_{i}^{\prime}+P E_{i}=\not K E_{f}+P E_{f} & \\
P E_{i}=P E_{f} & \\
m_{A} g h_{i}+m_{B} g \hbar_{i}=\left(m_{A+B}\right)(g)(h) & \\
(m)_{A}(\phi)\left(h_{i}\right)=(2 m)(g)\left(h_{f}\right) & \\
\frac{1}{2} h_{i}=h_{f}
\end{array}
$$

FIG. 4. Example solution by a student who applied the CM principle to an incorrect situation. 
to articulate this idea only after the discussion with the researcher during the interview.

Moreover, it is possible that the concept of the "infinitesimal" time before and after the collision involved in the $\mathrm{CM}$ principle was very challenging for the students. If students did not realize that momentum of the two putty system was conserved only immediately before and after the collision, they were likely to apply the CM principle in incorrect situations, as presented in Table XI, category CM_B. In all the interviews, students were asked explicitly to identify the situation in which their CM principle was applied by describing what their variables $v_{i}$ and $v_{f}$ in the $\mathrm{CM}$ equation refer to. They were sometimes given the following choices to help them articulate their answers: their CM principle was applied (1) from the very beginning to the very end, (2) from right before the collision to right after the collision, and (3) somewhere in between (1) and (2). During the interview, one student responded in the following manner.

Student B: Wouldn't they .... Wouldn't the total momentum for the system be the same throughout? Or would [it] not be?

Researcher: What do you think?

Student B: I think it would be.

Researcher: OK. So ... why?

Student B: Um ... just because from what I heard for the conservation of momentum, from what I've been told about, momentum is conserved throughout ... Uh ... yea, no matter what time it is, whether its $t$ [equals] zero or $t$ [equals] infinity, the momentum should be conserved throughout.

Researcher: OK. Do you remember when your professor taught you about conservation of momentum, did he or she say when should that principle be applicable?

Student B: Um ... I believe ... it may have been not applicable for inelastic collision but I don't really remember. And I believe it [the solution to the snowboard problem] says that this was inelastic ... Um ... I believe you would use it [conservation of momentum] more in elastic collision than you would in inelastic collision.

The dialogue above suggests that when the student exploited the CM principle to solve the quiz problem, he did not carefully examine the applicability of the CM principle by considering whether or not there was an external force acting on the system, and he did not notice that the momentum is only conserved from immediately before to immediately after the collision. In fact, the interaction between the researcher and the student suggests that it is very likely that the student did not know how to correctly examine the applicability of the CM principle.
When the researcher asked him about the situations in which the CM principle could be applicable, the student discussed the applicability incorrectly based upon whether the collision was elastic or inelastic. Findings from our study suggest that more scaffolding supports may be required to help students deal with this issue and acquire a better understanding of the situation in which the $\mathrm{CM}$ principle can be applied.

Another important student difficulty that hinders students from solving the putty problem correctly may be that the students may not necessarily understand the role of inelastic collision for mechanical energy conservation. For example, for students who made the mistakes CME_1 and CME_2 in Tables XI and XII, it is likely that they did not realize that during the inelastic collision some mechanical energy would be transformed into heat or other forms of energy and therefore the mechanical energy of the system was not conserved. During the interviews, when the students were asked about the difference between elastic and inelastic collisions, most students discussed the difference between two collisions by some surface feature such as (i) whether the object keeps its original shape and/or (ii) whether the two objects move apart or become one after the collision, instead of mentioning the definition of an elastic (inelastic) collision as a collision in which mechanical energy is (is not) conserved. When the researcher asked them to discuss further the specific principles that were applicable or not applicable in the elastic and inelastic collisions (e.g., by directly guiding them to consider the principles of CME and $\mathrm{CM}$ ), many of them did not know the correct answer and were unable to make up their mind. Quotes from two students are listed below. This finding suggest that even if students could recognize the inelastic collision process involved in the putty problem, they did not necessarily understand that the mechanical energy was not conserved throughout the whole process. More help may be required to help students in solving the putty problem correctly.

Researcher: Do you remember if this collision is an elastic or inelastic collision?

Student C: Uh. Yea. Elastic means that they come together and then they can retain their shape, right? like ... when they hit together, then when they come ap[apart] ... they can come apart, and so be the same shape as they were when they came into collision. And then ... and ... inelastic forces they hit and they are one object.

Researcher: do you mean they stick...

Student C: Yea they stick together and they stick together throughout the system.

Researcher: Ok. So do you still remember which principle is applicable during the collision process and which is not? I mean, is there any difference between these two kinds of collision? 
Student C: Uh ... I mean ... there is. I just ... I just don't know.

Researcher: How about ... let's think about the momentum?

Student C: Yea, it would be the difference between ... like ... conserves momentum and [does] not conserves momentum? Would that be it?

Researcher: What do you think?

Student C: I . . I . . I I actually don't know.

Researcher: Ok. So ... momentum is conserved in both kinds of collisions.

Student C: Oh, really?

Researcher: Yea.

Student $C: O k$.

Researcher: So do you still remember anything about energy?

Student C: I do. I ... I remember like ... kinetic energy equ[equation] ... uh ... conservation of energy equation, just cause that was what we were taught a lot about. And then, we are taught a little bit about momentum, but ... it shoots off very quickly. And I haven't taken physics before, so it's all new to me. So

Researcher: Yea, I understand that. Don't feel bad ... I mean ... we know the [CM] principle is very difficult for lots of students.

Student $C: O K$.

Researcher: So ... um ... you are not quite sure whether energy is conserved during ...

Student C: between elastic and inelastic?

Researcher: Uh huh. Do you remember anything about that?

Student $C:$ Uh ... energy is conserved in a ... inelastic collision? Or? Uh ... Let me think about this.

Researcher: Yea, take your time.

Student C: Uh ... Uh ... I guess energy is ... conserved in inelastic collision? Yeah, I think so.

Researcher: So is energy conserved in an elastic collision?

Student C: Uh ... is energy conserved in an elastic collision ...

[silence]
Student C: Uh ... I attempt to say no. But at the same time I don't think so. I don't think it's yes. I'm going to go with no because once you hit it, like you like for this object, when the one ball hits the other ball, and if it were inelastic, they come together, and all the energies is gonna fall in with them. Whereas if the one hits, this one is still on the, like going up a little bit but this one is also going up, so ... uh ... I guess energy is ... I'm going to go with energy is conserved in both.

Student F: There's one where you can use conservation of momentum and energy. And there's another one where you can use one of those. But I don't remember which one [elastic or inelastic] it goes to and which one [CM or CME] works.

Not being able to figure out the three-step nature of the quiz problem and mistakenly using the CME principle only once (in processes involving the inelastic collision such as those shown in groups CME_1 and CME_2 in Table XI) were the major difficulties many students had. Therefore, one may expect that intervention 3, which involves an additional hint about "using the conservation of mechanical energy twice," may be of great help to the students. However, the quantitative data obtained from the two introductory physics courses (see, for example, Tables VII, VIII, and XII) suggest that this intervention did not help students significantly. The same phenomenon was observed in the interview as well. Figure 5 presents the solution of a student who was subjected to intervention 3 during the think aloud interview. Initially, he did not appear to pay much attention to the additional hint about "using the conservation of mechanical energy twice" and in his work CME was used only once. After he had tried the putty problem on his own to the best of his knowledge (shown in Fig. 5), the researcher reminded him that the snowboard problem was a two-part problem and the putty problem was a three-part problem in which we had to use the CME principle twice. However, this reminder did not help, either. He still thought that the first step in solving the putty problem was to use the CM principle similar to what he did in his earlier work. He felt that the putty problem must be solved by using CM first, followed by the use of CME twice. He incorrectly interpreted that it was the last CME part that would lead him to express $h_{f}$ in terms of $h_{o}$ that he missed in his original work, but he struggled greatly and did not know what to do with it. This interview suggests that some students might interpret the instruction of "using CME twice" in a different way than was intended, which could be one possible reason why providing the additional instruction that might be considered an additional hint by the experts in intervention group 3 did not work as intended for the students.

Overall, the data from the two introductory courses as well as the interviews suggest that in this analogical problem solving and transfer activity, although students may be 


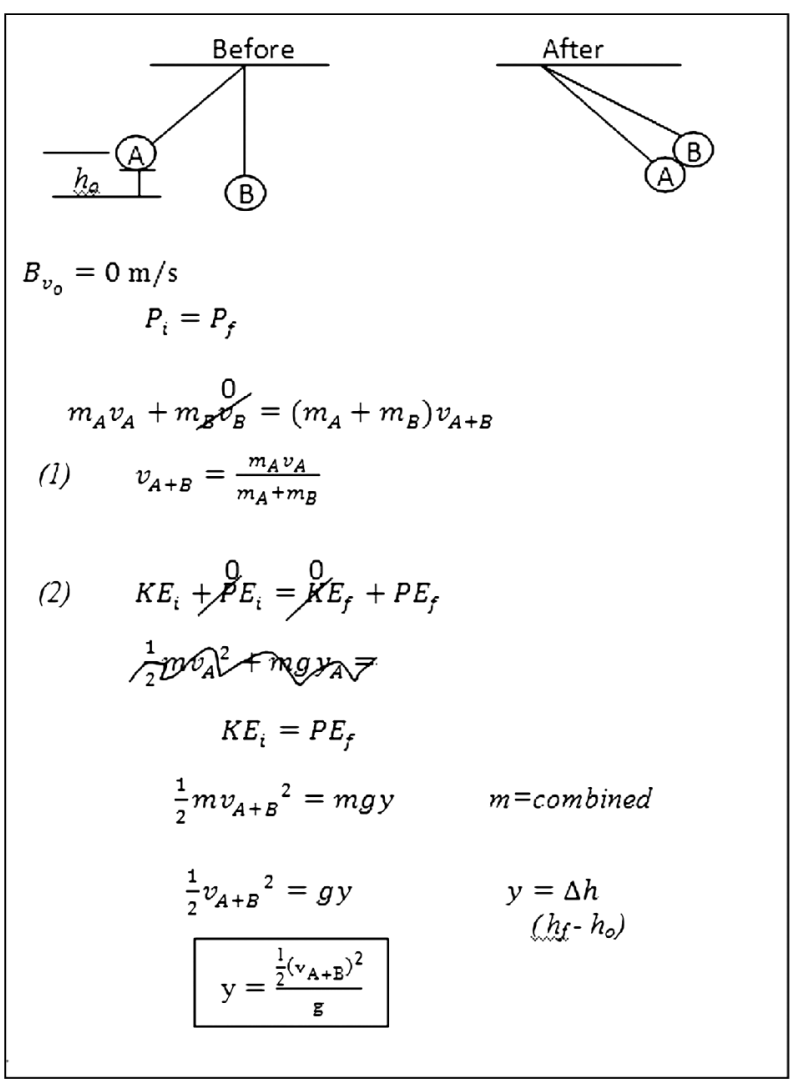

FIG. 5. Students A's answer to the putty problem.

able to recognize the similarity between the isomorphic problems in terms of the principles involved, the solution to the snowboard problem does not necessarily help them figure out the structure of the three-part putty problem and apply the principles correctly. In general, our research suggests that more algebra-based students benefited from the solved problem provided than the calculus-based students in the sense that the average scores in the intervention groups are significantly better than that in the comparison group. As shown in Table XII, many algebra-based control group students simply had no clue about how to solve the putty (transfer) problem when no scaffolding was provided. Some of them invoked the 1D kinematics equations and were not able to go far after that. Without additional support, some of them simply wrote down some potentialenergy-like or kinetic-energy-like terms separately without writing any equation. (These mistakes were all classified in category CME_5 "other structural mistake" in Table XII.) The fact that with the solved problem provided most of the algebra-based students were able to invoke either one or both of the correct principles (which they were not able to do when asked to solve the problem on their own) is the main reason why all three intervention groups performed significantly better than the comparison group in the algebra-based courses. However, the not-so-high absolute scores (around 44\%-54\%) after the scaffolding also reflect the fact that the algebra-based students were not necessarily able to apply the relevant principle correctly. For the calculus-based course, on the other hand, students typically were able to invoke the relevant principle(s) even without being provided the solved problem. The main difficulty for the calculus-based students therefore lies in how to proceduralize these principles in an appropriate manner. As we found in this study, our current scaffolding support provided (i.e., the solution to the snowboard problem together with different interventions implemented) was not effective in this regard; the difficulty of applying the principles correctly still remained even after they received the scaffolding.

\section{B. Effect of additional scaffolding (beyond those used in the two introductory physics courses) as suggested by the interviews}

As mentioned earlier, one important goal of the interviews was to examine the possible additional scaffoldings (i.e., scaffoldings beyond those used in the two introductory physics courses) that may help the students solve the quiz (transfer) problem correctly. The different scaffoldings that were tried in the interviews to help students solve the problem successfully included the following.

(i) Directing students' attention to the fact that energy is not conserved in an inelastic collision.

(ii) Directing students' attention to the fact that the CM principle is only valid right before to right after the collision.

(iii) Telling students explicitly that the problem can be decomposed into three parts.

(iv) Helping students learn how to solve a simpler twostep problem (the bridging two-block problem) first.

As noted earlier, depending on the prior knowledge and difficulties students had, different students received different levels of additional scaffolding in the interviews to help them solve the quiz problem correctly. A detailed list of the additional scaffoldings that each interviewed student received as well as the effects of these scaffoldings can be found in Appendix D of the Supplemental Material [58]. (Student B was not included in Appendix D because there was not enough time in the interview to provide him with additional scaffoldings to help him solve the putty problem correctly.)

In general, these additional scaffoldings are provided with the intent to help students figure out the three-step nature of the quiz problem and the situation in which the CM or CME principle should be applicable, since these are the greatest difficulties students had as described in the previous section. Overall, the interviews suggest that simply directing students to go back to the solved problem and to explicitly identify the situation in which each physical principle is applicable in the solved problem did not help students much partly because they did not necessarily ask 
themselves why this principle was applicable here, and more importantly, why it was not applicable to some other situations. As noted earlier, even if students were able to describe that the CM principle was applicable in the solved problem from right before to right after the collision, they may still draw from their previous experience that the CM principle was always valid from $t=0$ to $t=\infty$. Thus, in the putty problem, they were likely to use the CM principle throughout.

The interviews suggest that a good way to help students learn the applicability of the CM and CME principles from the solved problem provided is to use the solved problem to guide students to contemplate deeply (1) why and how each principle is applied in the way shown in the solved situation and, (2) more importantly, why those principles are not applicable in other situations (e.g., why is it inappropriate to apply the CME or CM principle throughout the whole process?). For example, the interviews with students $\mathrm{E}$ and F suggest that, if a detailed discussion with the students about the solved problem was carried to help students learn deeply (e.g., about the fact that when the snowboard goes up the ramp, the net external force acting on the system is not zero and the velocity of the snowboard keeps changing, so the momentum could not be conserved), students are likely to realize themselves that in the quiz (transfer) problem, the momentum principle should be applicable only between right before and right after the collision. In comparison, in the interviews with other students, in which no similar discussion ensued, students were not be able to figure out how the CM principle should be involved to solve the putty problem unless an explicit instruction telling them to apply the CM principle only right before and right after the collision was provided. A list of important issues for discussion related to the solution of the snowboard problem which may be beneficial for the students include the following. (a) When is the CM principle applicable? (The instructors may give students several choices including "from immediately before to immediately after the collision," "from the very beginning to the very end when the person reaches the maximum height," etc.) (b) Why is the CM principle not applied elsewhere in the problem? Could it be applicable elsewhere in the problem? Why or why not? (c) From where to where in the problem is the CME principle applicable? Why do we apply CME only in this part of the problem? Can we go beyond that and apply it throughout the whole process in the problem? (d) What is an appropriate system for applying each of these principles? Such questions could keep students more actively involved in the learning process while working on the solved example and help them benefit more from the self-explanation process as discussed in the study of Chi et al. [62].

However, in order to solve the quiz (transfer) problem correctly, not only do students need to understand that neither the CME nor the CM principle could be applied throughout the whole process, they also must have a clear picture of the three-step structure of the quiz problem as well as how each subproblem is connected to each other. In order to help students with these issues, in the interviews with students $\mathrm{E}$ and $\mathrm{F}$, we also examined the effect of introducing a new problem (the "two-block problem" shown in Appendix C of the Supplemental Material [58]) as a bridging problem to help students solve the putty problem involving three parts. This new two-block problem consists of only two steps: an object going down, colliding, sticking, and moving together with another object on the horizontal part of the track. This bridging problem is a two-step problem which is very similar to the solved problem except that the processes are reversed. After students $\mathrm{E}$ and $\mathrm{F}$ realized how to solve the new bridging problem correctly, we then asked them to take advantage of what they learned from these two problems to solve the three-step putty problem. (A detailed description of the interventions students $\mathrm{E}$ and $\mathrm{F}$ received can be found in Table V.) We hypothesized that after the students understand how to solve the snowboard problem and the twoblock bridging problem, they will have a better idea of the three processes involved in the putty problem and they may be able to construct a holistic picture of how the different subproblems should be connected.

Indeed, the interviews suggest that the additional scaffoldings provided to students $\mathrm{E}$ and $\mathrm{F}$ [which involves (a) a discussion guiding students to contemplate why and how each principle was applied in the way shown in the solution and not in other situations and (b) the "two-block" problem] are very likely to benefit students. In the interviews with students $\mathrm{A}, \mathrm{C}$, and $\mathrm{D}$, in which the bridging problem was not provided and we did not use the solved problem to discuss the issues related to the applicability of the CM or CME principle, we found that significant help from the researcher is usually required to help students solve the putty (transfer) problem correctly. For example, in addition to the reminder that the CME principle could not be applied in processes involving the inelastic collision, students $\mathrm{C}$ and $\mathrm{D}$ required an explicit instruction in "applying the $\mathrm{CM}$ principle only right before and right after the collision in the putty problem" as well as other assistance (such as guidance about how to connect the three subproblems to express $h_{f}$ in terms of $h_{o}$ or an instruction showing how to solve a particular subproblem involving CME) to help them solve the putty problem. Similarly, student A was not able to figure out the correct solution to the putty problem unless the whole problem was broken into subproblems in which the target in each subproblem was specified and other necessary scaffolding supports were provided.

On the other hand, in the interviews with students $\mathrm{E}$ and $\mathrm{F}$, we found that after enough discussion with the students and explanation about why and how each principle was applied in the way shown in the solution to the snowboard 
problem, and after the students understood how to solve the bridging problem (i.e., the two-block problem) correctly following the discussion, they were able to take advantage of what they learned from the two problems (snowboard problem and the two-block problem) and correctly transfer their learning to solve the putty problem on their own. The critical scaffolding provided was the help in recognizing the similarities between the snowboard problem and the two-block problem and understanding how to solve the two-block problem correctly. One student required more help in understanding that something going down in the two-block problem is in principle the same as something going up in the solved snowboard problem. However, after students $\mathrm{E}$ and $\mathrm{F}$ recognized this similarity, understood that the mechanical energy of the system is not conserved during the inelastic collision, and realized why $\mathrm{CM}$ is not valid throughout the whole process in the snowboard problem or the two-block problem, the putty problem was not as difficult for them as for other students. After the scaffolding described above, without help from the researcher, students $\mathrm{E}$ and $\mathrm{F}$ themselves recognized that the putty problem should be split into three subproblems and CME, CM, and CME should be applied to the three consecutive subproblems, respectively. Although the fact that the final velocity in one subproblem becomes the initial velocity in the next subproblem was somewhat frustrating for one student, both of them had a clear picture of the whole solution process and were able to solve the quiz problem correctly on their own.

\section{CONCLUSION AND FUTURE OUTLOOK}

In this study, we found that it is very challenging for introductory physics students to transfer what they learned in the two-step snowboard problem to solve the three-step putty problem. In the calculus-based course, when students were asked to learn from the solution to the snowboard problem provided and take advantage of what they learned from the snowboard problem to solve the putty problem which is isomorphic, they did not outperform their peers in the comparison group, who were not provided with any scaffolding support to solve the putty problem. Although students in the algebra-based course who received the scaffolding with the solved problem significantly outperformed their peers in the comparison group, their average scores-which fell in the range of only $44 \%-54 \%$ suggest that there is still much room for improvement. Findings revealed that the greatest difficulty students had in transferring was in applying the physics principles learned from the solved problem to the new situation presented in the quiz problem in an appropriate way. Even though the solved problem could help students invoke the relevant principles in the quiz problem (which is the main reason that in the algebra-based course students who received the scaffolding with the solved problem outperformed students in the comparison group, most of whom had no clue how to go about solving the putty problem), many students did not have a clear plan for how those principles should be applied to solve the putty problem. They did not understand how to decompose the putty problem into suitable subproblems and they sometimes combined several processes into one, applied the physics principles in inappropriate situations, or applied the principles correctly but did not discern their relevance to the final answer (target variable). For calculus-based students, many of them were able to invoke the relevant principles even without learning from the solved problem, and the greatest difficulty was in applying the principles correctly. The scaffolding supports provided did not help them significantly. Algebra-based students generally had even more difficulty in applying the principles correctly.

In a previous study, students were asked to perform analogical problem solving between a solved problem and an isomorphic quiz problem, both of which were two-step problems. Comparing students' ability to transfer in this study to that in the previous study, we find that even though the problems in the previous study required the application of Newton's second law in the nonequilibrium situation, which is typically challenging for students, on average students displayed better transfer for the case discussed in the previous study. The fact that in our current study the solved problem provided was a two-step problem whereas the targeted problem was a three-step problem made the transfer very challenging. With the existence of an additional step in the quiz problem, students could no longer map the solved problem directly to the quiz problem. They had to learn from the solved example and understand the circumstances for which each principle is applicable, so as to be able to systematically decompose the transfer problem into appropriate subproblems (that can be dealt with one at a time with a single principle). The interviews suggest that students often superficially mapped the principles employed in the solved problem to the quiz problem without necessarily understanding the governing conditions underlying each principle or examining the applicability of the principles in the new situation in an in-depth manner.

Our findings suggest that determining the situations in which the CM and CME principles are applicable is very challenging for students despite providing them with a solved problem and more scaffolding is required in order to help students perform better on the transfer problem. However, the idea is not to spoon-feed them; rather, the dimensions of efficiency and innovation as described in the model of Schwartz, Bransford, and Sears are both important for transfer [26,43]. Students should be actively engaged in the analogical reasoning process themselves and in reconstructing, organizing, and extending their knowledge structure. It is possible that if students are guided to think about the solution in more depth and contemplate the applicability of various principles in the 
solution carefully, they are more likely to benefit from the solved problem provided.

One possible way to guide students' self-explanation toward this goal is suggested in the interview part of this study and can be investigated in-depth in the future. In particular, if suitable questions are designed about the applicability of the principles used in the solved problem, and before students solve the quiz problem, they are asked to justify why those physics principles are applicable in the situations in the solved examples, but not in other situations, they may learn better from the solved problem provided.

Interviews also suggest that another strategy that may assist students in figuring out the three-step structure of the putty problem is to add a bridging problem in this analogical problem-solving activity. For example, after students learn from the solved snowboard problem, they can be asked to solve a bridging problem (such as the two-block problem discussed in the interview section) first before they solve the putty problem. In the interviews, we found that if the students understood why and how the CM and CME principles are applicable in the snowboard problem and the two-block problem, why the CM principle is not valid for other subproblems before or after the collision, and why the CME principle is not applicable throughout the whole process in the two problems, they were likely to solve the putty problem correctly on their own without help. The bridging problem can be further tested in future studies with a larger group of students to examine its effect in large classes.

Additional scaffoldings may also be designed and tested in future studies to help students with specific difficulties in problem solving. For example, if the students are unsure about whether they should use the CM and CME principles for the inelastic collision process, instead of simply telling them the correct answer, an intervention could deliberately direct students to contemplate both the momentum and mechanical energy of the system right before and right after the collision (for example, by asking them to compute the speeds and kinetic energies at these two instances and compare whether their results are consistent with the predictions they made for both conservation laws). It is possible that by doing so, e.g., in the problem discussed in this study, they will be more likely to understand that some mechanical energy will be transformed into other forms of energy and they cannot simply set the initial potential energy of one putty equal to the final potential energy of both putties together without contemplating the inelastic collision process in between. Future studies could also explore transfer between a solved problem and an isomorphic transfer problem in situations where two students work together on making sense of the solved problem and on transferring their learning from one situation to another.

In summary, deliberately using an isomorphic workedout example to help students transfer what they learned from one context to another can be a useful tool to help students understand the applicability of physics principles in diverse situations and develop a coherent knowledge structure of physics. For introductory students, such wellthought-out activities could provide a model for effective physics learning since the idea of looking at deep similarities beyond the surface features is enforced throughout these activities. Since our study suggests that it can be challenging for students to correctly apply what they learned from a two-step problem to solve a three-step problem, more scaffolding supports that are commensurate with students' prior knowledge may be required to help them realize the structure of the solution and to learn from the solved example effectively. It can be beneficial if the importance of looking for governing conditions underlying each principle and examining the applicability of the physics principles in the new situation in an in-depth manner are consistently explained, emphasized, demonstrated, and rewarded by the instructors. It is possible that students will become more facile at the analogical problem-solving processes and be able to transfer their learning from one situation to another if suitable scaffolding, practice. and feedback are constantly provided to them throughout the whole course.
[1] M. T. H. Chi, P. J. Feltovich, and R. Glaser, Categorization and representation of physics problems by experts and novices, Cogn. Sci. 5, 121 (1981).

[2] B. Eylon and F. Reif, Effect of knowledge organization on task performance, Cognit. Instr. 1, 5 (1984).

[3] J.H. Larkin and F. Reif, Understanding and teaching problem-solving in physics, Eur. J. Sci. Educ. 1, 191 (1979).

[4] D. Hammer, Student resources for learning introductory physics, Am. J. Phys. 68, S52 (2000).

[5] E.F. Redish, J. M. Saul, and R. N. Steinberg, Student expectations in introductory physics, Am. J. Phys. 66, 212 (1998).
[6] E. F. Redish, R.E. Scherr, and J. Tuminaro, Reverseengineering the solution of a "simple" physics problem: Why learning physics is harder than it looks, Phys. Teach. 44, 293 (2006).

[7] F. Reif, Teaching problem solving-A scientific approach, Phys. Teach. 19, 310 (1981).

[8] F. Reif, Millikan Lecture 1994: Understanding and teaching important scientific thought processes, Am. J. Phys. 63, 17 (1995).

[9] D. Maloney, Research in problem solving: Physics, in Handbook of Research on the Teaching and Learning of Science, edited by D. Gabel (MacMillan, New York, 1994). 
[10] P. Hardiman, R. Dufresne, and J. Mestre, The relation between problem categorization and problem solving among experts and novices, Mem. Cogn. 17, 627 (1989).

[11] F. Reif and J. I. Heller, Knowledge structure and problem solving, Educ. Psychol. 17, 102 (1982).

[12] A. Schoenfeld and D. J. Herrmann, Problem perception and knowledge structure in expert and novice mathematical problem solvers, J. Exp. Psychol. Learn. Mem. Cogn. 8, 484 (1982).

[13] D. Bobrow and D. Norman, Some principles of memory schemata, in Representation and Understanding: Studies in Cognitive Science, edited by D. Bobrow and A. Collins (Academic, New York, 1975), pp. 131-149.

[14] P. W. Cheng and K. J. Holyoak, Pragmatic reasoning schemas, Cogn. Psychol. 17, 391 (1985).

[15] K. Johnson and C. Mervis, Effects of varying levels of expertise on the basic level of categorization, J. Exp. Psychol. Gen. 126, 248 (1997).

[16] P. Johnson-Laird, Psychology of Reasoning, Structure and Content (Harvard University Press, Cambridge, MA, 1972).

[17] J. Larkin, Cognition of learning physics, Am. J. Phys. 49, 534 (1981).

[18] S. Marshall, Schemas in Problem Solving (Cambridge University Press, New York, 1995).

[19] R. Dufresne, J. Mestre, T. Thaden-Koch, W. Gerace, and W. Leonard, Knowledge representation and coordination in the transfer process, in Transfer of Learning from a Modern Multidisciplinary Perspective, edited by J.P. Mestre (Information Age Publishing, Greenwich, CT, 2005), pp. 155-215.

[20] M. Ferguson-Hessler and T.d. Jong, Studying physics texts: Differences in study processes between good and poor performers, Cognit. Instr. 7, 41 (1990).

[21] J. Larkin, Skilled problem solving in physics: A hierarchical planning model, J. Struct. Learn. 6, 271 (1980).

[22] J. Larkin, Skilled problem solving in physics: A hierarchical approach, J. Struct. Learn. 6, 121 (1980).

[23] M. Bassok and K. Holyoak, Interdomain transfer between isomorphic topics in algebra and physics, J. Exp. Psychol. Learn. Mem. Cogn. 15, 153 (1989).

[24] K.E. Gray and N.S. Rebello, Transfer between paired problems in an interview, AIP Conf. Proc. 790, 157 (2005).

[25] J. Mestre, Probing adults' conceptual understanding and transfer of learning via problem posing, J. Appl. Dev. Psychol. 23, 9 (2002).

[26] J. D. Bransford and D. Schwartz, Rethinking transfer: A simple proposal with multiple implications, Rev. Res. Educ. 24, 61 (1999).

[27] J. Lobato, How design experiments can inform a rethinking of transfer and vice versa, Educ. Res. 32, 17 (2003).

[28] J. Lobato, Alternative perspectives on the transfer of learning: History, issues, and challenges for future research, J. Learn. Sci. 15, 431 (2006).

[29] J. Mestre, in Transfer of Learning from a Modern Multidisciplinary Perspective (Ref. [19]).

[30] D. Hammer, A. Elby, R. Scherr, and E.F. Redish, Resources, framing and transfer, in Transfer of Learning from a Modern Multidisciplinary Perspective (Ref. [19]).

[31] P. Adey and M. Shayer, An exploration of long-term far-transfer effects following an extended intervention program in the high school science curriculum, Cognit. Instr. 11, 1 (1993).

[32] D. K. Detterman and R. J. Sternberg, Transfer on Trial: Intelligence, Cognition and Instruction (Ablex, Norwood, NJ, 1993).

[33] D. J. Ozimek, P. V. Engelhardt, A. G. Bennett, and N. S. Rebello, Retention and transfer from trigonometry to physics, AIP Conf. Proc. 790, 173 (2005).

[34] N. S. Rebello, L. Cui, A. G. Bennett, D. A. Zollman, and D. J. Ozimek, Transfer of learning in problem solving in the context of mathematics and physics, in Learning to Solve Complex Scientific Problems, edited by D. Jonassen (Lawrence Erlbaum Associates, New York, 2007).

[35] N.S. Rebello and D.A. Zollman, Dynamic transfer: A perspective from physics education research, in Transfer of Learning from a Modern Multidisciplinary Perspective (Ref. [19]).

[36] C. Singh, Transfer of learning in quantum mechanics, AIP Conf. Proc. 790, 23 (2005).

[37] M. Gick and K. Holyoak, Schema induction and analogical transfer, Cogn. Psychol. 15, 1 (1983).

[38] A. Brown, Analogical learning and transfer: What develops?, in Similarity and Analogical Reasoning, edited by S. Vosniadou and A. Ortony (Cambridge University Press, New York, 1989), pp. 369-412.

[39] D. Genter and C. Toupin, Systematicity and surface similarity in the development of analogy, Cogn. Sci. 10, 277 (1986).

[40] J. Mestre, Implications of research on learning for the education of prospective science and physics teachers, Phys. Educ. 36, 44 (2001).

[41] L. Novick, Analogical transfer, problem similarity and expertise, J. Exp. Psychol. Learn. Mem. Cogn. 14, 510 (1988)

[42] C. Singh, Assessing student expertise in introductory physics with isomorphic problems. II. Effect of some potential factors on problem solving and transfer, Phys. Rev. ST Phys. Educ. Res. 4, 010105 (2008).

[43] D. Schwartz, J. Bransford, and D. Sears, Efficiency and innovation in transfer, in Transfer of Learning: Research and Perspectives, edited by J. Mestre (Information Age Publishing, Greenwich, CT, 2005), pp. 1-51.

[44] K. Holyoak, The pragmatics of analogical transfer, in The Psychology of Learning and Motivation, edited by G. Bower (Academic, New York, 1985), Vol. 19.

[45] R. J. Sternberg, Component processes in analogical reasoning, Psychol. Rev. 84, 353 (1977).

[46] S.-Y. Lin and C. Singh, Using isomorphic problems to learn introductory physics, Phys. Rev. ST Phys. Educ. Res. 7, 020104 (2011).

[47] R. Duit, On the role of analogies and metaphors in learning science, Sci. Educ. 75, 649 (1991).

[48] S. K. Reed, G. W. Ernst, and R. Bannerji, The role of analogy in transfer between similar problem states, Cogn. Psychol. 6, 436 (1974).

[49] B. Ross, Distinguishing types of superficial similarities: Different effects on the access and use of earlier problems, J. Exp. Psychol. Learn. Mem. Cogn. 15, 456 (1989).

[50] M. A. Shapiro, Analogies, visualization and mental processing of science stories, in Proceedings of the 
Conference of the Information Systems Division of the International Communication Association, 1985 (1985).

[51] N. S. Podolefsky and N. D. Finkelstein, Analogical scaffolding and the learning of abstract ideas in physics: An example from electromagnetic waves, Phys. Rev. ST Phys. Educ. Res. 3, 010109 (2007).

[52] M. A. Shapiro, in Communication Yearbook, edited by M. McLaughlin (Sage, Beverly Hills, CA, 1988), Vol. 9.

[53] J. R. Hayes and H. A. Simon, Psychological differences among problem isomorphs, in Cognitive Theory, edited by N. J. Castellan, D. B. Pisoni, and G. R. Potts (Lawrence Erlbaum, Hillsdale, NJ, 1977).

[54] K. Kotovsky, J. R. Hayes, and H. A. Simon, Why are some problems hard? Evidence from Tower of Hanoi, Cogn. Psychol. 17, 248 (1985).

[55] H. A. Simon and J. R. Hayes, The understanding process: Problem isomorphs, Cogn. Psychol. 8, 165 (1976).

[56] C. Singh, Assessing student expertise in introductory physics with isomorphic problems. I. Performance on nonintuitive problem pair from introductory physics, Phys. Rev. ST Phys. Educ. Res. 4, 010104 (2008).

[57] C. Singh and D. Rosengrant, Multiple-choice test of energy and momentum concepts, Am. J. Phys. 71, 607 (2003).

[58] See Supplemental Material at http://link.aps.org/ supplemental/10.1103/PhysRevSTPER.9.020114 for the quiz problem, the solved problem, the full instruction provided to intervention group 3 , and the additional scaffolding students received in the interview studies.

[59] V. Aleven, K. R. Koedinger, and K. Cross, Tutoring answer explanation fosters learning with understanding, in Proceedings of the Conference on Artificial Intelligence in Education, Open Learning Environments: New Computational Technologies to Support Learning, Exploration, and Collaboration (AIED-99) (IOS Press, Amsterdam, 1999), pp 199-206.

[60] R. K. Atkinson, S. J. Derry, A. Renkl, and D. Wortham, Learning from examples: Instructional principles from the worked examples research, Rev. Educ. Res. 70, 181 (2000).
[61] M. Chi, Self-explaining expository texts: The dual processes of generating inferences and repairing mental models, in Advances in Instructional Psychology, edited by R. Glaser (Erlbaum Associates, Mahwah, NJ, 2000), pp. 161-238.

[62] M. Chi, M. Bassok, M. W. Lewis, P. Remann, and R. Glaser, Self-explanations: How students study and use examples in learning to solve problems, Cogn. Sci. 13, 145 (1989).

[63] E. Yerushalmi, A. Mason, E. Cohen, and C. Singh, Effect of self diagnosis on subsequent problem solving performance, AIP Conf. Proc. 1064, 53 (2008).

[64] We have also examined the results when the overall performance of each intervention group is represented by a weighted mean of students' performance from three different levels of expertise (i.e., each average is weighted by the corresponding number of students in that group with a particular level of expertise). Our analysis shows that the results are the same regardless of whether the overall performance is represented by a weighted mean or an unweighted mean. For simplicity, in this paper we will show only the data for the performance with an unweighted mean.

[65] Although the middle group in the algebra-based course has a lower average score on the solved problem, examination of students' performance in details indicates that this score may have been affected by a few students with extreme scores since there is a total of only 11 students in this group. Our data show that two of the students in this group received a score of 0 and 3 on the solved problem, respectively, while all the other students received a score of 5 or more. If those two students were not included, the average score on the solved problem would be 8 for the middle group and 8.7 for the "all."

[66] E. Yerushalmi, E. Cohen, A. Mason, and C. Singh, What do students do when asked to diagnose their mistakes? Does it help them? I. An atypical quiz context, Phys. Rev. ST Phys. Educ. Res. 8, 020109 (2012).

[67] E. Yerushalmi, E. Cohen, A. Mason, and C. Singh, What do students do when asked to diagnose their mistakes? Does it help them? II. A more typical quiz context, Phys. Rev. ST Phys. Educ. Res. 8, 020110 (2012). 